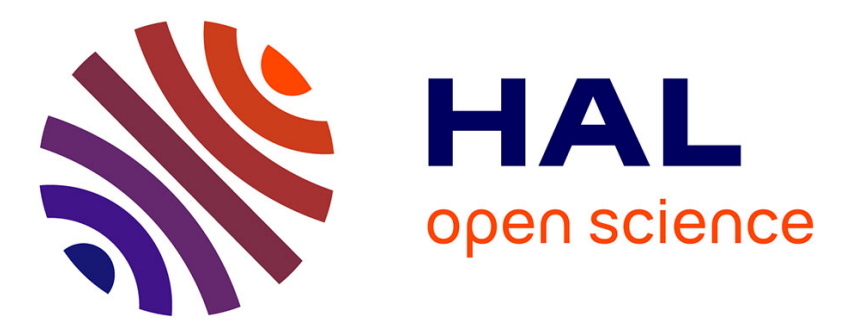

\title{
Requirement for endoderm and FGF3 in ventral head skeleton formation
}

Nicolas B. David, Laure Saint-Etienne, Michael Tsang, Thomas F Schilling, Frédéric M. Rosa

\section{> To cite this version:}

Nicolas B. David, Laure Saint-Etienne, Michael Tsang, Thomas F Schilling, Frédéric M. Rosa. Requirement for endoderm and FGF3 in ventral head skeleton formation. Development (Cambridge, England), 2002. hal-02362126

\section{HAL Id: hal-02362126 \\ https://hal.science/hal-02362126}

Submitted on 15 Nov 2019

HAL is a multi-disciplinary open access archive for the deposit and dissemination of scientific research documents, whether they are published or not. The documents may come from teaching and research institutions in France or abroad, or from public or private research centers.
L'archive ouverte pluridisciplinaire HAL, est destinée au dépôt et à la diffusion de documents scientifiques de niveau recherche, publiés ou non, émanant des établissements d'enseignement et de recherche français ou étrangers, des laboratoires publics ou privés. 


\title{
Requirement for endoderm and FGF3 in ventral head skeleton formation
}

\author{
Nicolas B. David1, Laure Saint-Etienne ${ }^{1}$, Michael Tsang ${ }^{2}$, Thomas F. Schilling ${ }^{3, \dagger}$ and Frédéric M. Rosa ${ }^{1, \star, \dagger}$ \\ 1 U 368 INSERM, Ecole Normale Supérieure, 46, rue d' Ulm, F-75230 Paris Cedex 05, France \\ 2Laboratory of Molecular Genetics, National Institute of Child Health and Human Development, NIH, Bethesda MD, USA \\ ${ }^{3}$ Developmental and Cell Biology, University of California, Irvine CA 92697, USA \\ *Author for correspondence (e-mail: rosa@wotan.ens.fr) \\ tThese two authors contributed equally to this work \\ Accepted 27 June 2002
}

\section{SUMMARY}

The vertebrate head skeleton is derived in part from neural crest cells, which physically interact with head ectoderm, mesoderm and endoderm to shape the pharyngeal arches. The cellular and molecular nature of these interactions is poorly understood, and we explore here the function of endoderm in this process. By genetic ablation and reintroduction of endoderm in zebrafish, we show that it is required for the development of chondrogenic neural crest cells, including their identity, survival and differentiation into arch cartilages. Using a genetic interference approach, we further identify Fgf3 as a critical component of endodermal function that allows the development of posterior arch cartilages. Together, our results reveal for the first time that the endoderm provides differential cues along the anteroposterior axis to control ventral head skeleton development and demonstrate that this function is mediated in part by Fgf3.

Key words: Neural crest, Fate, Cartilage, Endoderm, Patterning, casanova

\section{INTRODUCTION}

The development of the vertebrate head skeleton represents an interesting paradigm to decipher the cellular and molecular bases of organogenesis. During head development, cephalic neural crest cells originate from mid- and hindbrain levels, migrate ventrally in a segmental fashion, and ultimately generate most of the head skeleton, as well as sensory neurons, glial and pigment cells and connective tissues (Le Douarin, 1982). During their migration, cephalic neural crest cells encounter segmented outpockets of endoderm budding from the wall of the pharynx, the pharyngeal 'pouches', and colonise the space between each pouch. Subsequently, neural crest cells, head mesoderm and endoderm interact to shape a series of complex elements, the pharyngeal arches, consisting of a core cartilage surrounded by pharyngeal epithelia, blood vessels, muscles and nerve ganglia (Trainor and Krumlauf, 2001). In addition to this cellular complexity, the pharyngeal region exhibits extensive patterning along the anteroposterior (AP) and dorsoventral (DV) axes, characterised, in particular, by the formation of different cartilage elements, nerve ganglia and muscles along these axes.

Epithelial-mesenchymal interactions are crucial for the proper development of the pharyngeal region. For instance, pharyngeal muscles are specified by an interaction between neural crest-derived ectomesenchyme and head mesoderm (Noden, 1983; Schilling et al., 1996a; Schilling and Kimmel, 1997). The pharyngeal ectoderm of the most anterior arch in the mouse produces signals, including FGF8, essential for the differentiation of neural crest cells into chondrocytes and for the DV patterning of cartilage elements within this arch (Trumpp et al., 1999). Similarly, Endothelin-1, expressed by both head paraxial mesoderm and pharyngeal epithelia, is crucial to the development of ventral pharyngeal arch cartilages (Kurihara et al., 1994; Miller et al., 2000). Epithelialmesenchymal interactions also appear critical for the proper AP patterning of the pharyngeal arches. Heterotopic transplantation of entire hindbrain/neural crest segments had suggested that mesenchymal neural crest cells were prepatterned along the AP axis before leaving the neuroectoderm to give rise to the different cartilages and bones of the arches. However, heterotopic transplantation of single neural crest cells or small cell groups have suggested rather that AP identity of neural crest cells can be reprogrammed under the influence of cues provided by the environment (Trainor and Krumlauf, 2001; Schilling et al., 2001). Furthermore, Hoxa2 misexpression at postmigratory stages has demonstrated that mandibular crest can be reprogrammed after migration (Pasqualetti et al., 2000).

Pharyngeal endoderm physically interacts with neural crest mesenchyme and might also play an important function in the control of neural crest fate and arch development. Probably because it is not easily accessible, however, its function in the pharyngeal region has been poorly defined. Extirpations of the most anterior (mandibular) pharyngeal endoderm or ectopic grafts of neural crest-derived ectomesenchyme with or without associated endoderm have been performed in amphibian, chick and mouse embryos (Graveson and Armstrong, 1987; Hall, 1980; Seufert and Hall, 1990). These experiments suggest that mandibular ectomesenchyme can only differentiate into 
cartilage when co-cultured or co-grafted with anterior (foregut, stomodeal or pharyngeal), but not with posterior (midgut or hindgut) endoderm. Whether or not pharyngeal endoderm is only involved in later stages of cartilage differentiation or has an earlier function in the control of neural crest fate and how this function is mediated at the molecular level is not understood at present. Pharyngeal endoderm also exhibits some degree of AP patterning, and recent evidence from grafts of foregut endoderm in chick suggests that this reflects a differential function of this tissue along the AP axis (Graham and Smith, 2001; Couly et al., 2002).

In vertebrates, endoderm and mesoderm are specified by Nodal-related signals, members of the transforming growth factor $\beta$ superfamily (Schier and Shen, 2000). Nodal activities are mediated by type I TGF $\beta$ receptors ALK4 and ALK7 in mammals and most likely by their relative, Taram-A (tar) in zebrafish (Aoki et al., 2002a; Reissmann et al., 2001; Yeo and Whitman, 2001). Activation of the Nodal pathway by expression of a constitutively active version of tar (tar*) leads to a respecification of early zebrafish blastomeres to an endodermal fate, consistent with the model that high levels of Nodal signalling are sufficient to direct cells to become endoderm (David and Rosa, 2001; Peyrieras et al., 1998). Downstream of nodal signalling, endoderm formation further involves the homeobox transcription factors Mixer/Bonnie and clyde (bon) and the recently identified Sox-related factor Casanova (cas) (Dickmeis et al., 2001; Kikuchi et al., 2001; Kikuchi et al., 2000). Zebrafish embryos in which Nodal signals are inactive develop neither endoderm nor mesoderm (Feldman et al., 1998) while, in contrast, bon or cas mutants form mesoderm but little or no endoderm (Alexander et al., 1999; Aoki et al., 2002b). In particular, cas mutants lack expression of all endodermal markers and derivatives. Interestingly, cas embryos are not rescued by activation of Nodal-related ligands or of the Tar cascade, consistent with its proposed function downstream. However, they provide a permissive environment for endoderm development since tar*activated wild-type blastomeres can autonomously restore endoderm formation when grafted into cas embryos (David and Rosa, 2001).

We have used cas and bon mutants to test whether or not pharyngeal endoderm is required for formation of the pharyngeal skeleton. We show that both mutants lack most of the ventral cartilage of the head skeleton and that these gene functions are required after cephalic neural crest migration, when crest normally contacts the endoderm. We fate map neural crest in cas and show that, in the absence of endoderm, cephalic neural crest cells remain as a cluster on the surface of the yolk sac and down regulate the expression of prechondrogenic markers. cas neural crest cells still have the ability to develop into cartilage when transplanted into wildtype embryos, showing that the cas gene is not required autonomously in neural crest cells but rather in their environment. Furthermore, endoderm can rescue head cartilage formation when reintroduced by grafting wild-type tar*injected cells into cas embryos. Finally, we implicate FGF in signalling from endoderm to neural crest by demonstrating that endodermal expression of FGF3 is specifically required for the formation of the posterior, branchial arches. Altogether, our results demonstrate a requirement for pharyngeal endoderm and FGF signalling in the control of head neural crest fates and cartilage induction, and identifies FGF3 as the first endodermal signal with an AP restricted function in the pharyngeal region.

\section{MATERIALS AND METHODS}

\section{Embryos}

Embryos were obtained from natural spawning of wild type (AB), heterozygous cas $^{\text {ta56 }}$, milte273 (Chen et al., 1996) or bon ${ }^{m 425}$ (Stainier et al., 1996) adult zebrafish. When indicated, embryos were dechorionated and incubated in SU5402 for 24 hours starting at the 15-somite stage (Shinya et al., 2001).

\section{Fate mapping of chondrogenic neural crest cells}

$10 \mathrm{kDa}$ DMNB-caged fluorescein $(5 \mathrm{mg} / \mathrm{ml})$ was injected into 2-cell stage embryos, then activated when embryos had reached the 5- to 7somite stage in a region encompassing the dorsal and lateral neural tube (Bally-Cuif et al., 2000; Girdham and O'Farrell, 1994). The location of the labelled cells was analysed at 28 hours either by visual inspection or by immunodetection of fluorescein.

\section{Grafting experiments}

Donor embryos were injected at the 4-cell stage with FITC-dextran. At the 10-somite stage, labelled premigratory neural crest were transplanted into wild-type hosts (David and Rosa, 2001; Schilling et al., 1996a). To restore endodermal derivatives in cas embryos, wildtype donors were injected with 80/1.2 pg GFP/Tar* RNA (or GFP alone as control) together with 120 pg nls-lacZ RNA or with $3 \% 10$ $\mathrm{kDa}$ dextran biotin. At the sphere stage, 10-20 donor cells were grafted to the margin of the progeny of cas/+ heterozygotes. When grafted a this stage, cells from control donors do not contribute efficiently to endoderm (Aoki et al., 2002b).

\section{Morpholino oligomer injections}

Morpholino oligomers obtained from Gene Tools Inc. were diluted in Danieaux solution. $f g f 3$-morpholino (Shinya et al., 2001; Phillips et al., 2001) was injected at the 1-cell stage or at the 16-cell stage at a concentration of $0.1 \mathrm{mM}$. $f g f 4$ was injected at varying concentrations up to $0.5 \mathrm{mM}$. fgf3-Mo: 5'-CATTGTGGCATGGAGGGATGTCGGC; fgf4-Mo: 5'-TTCTAAAAGGAGTTGAAGACACCG.

\section{Phenotypic analyses}

In situ hybridisation and immunohistochemistry were done following standard protocols (Hauptmann and Gerster, 1994). Probes and antibodies used were: $c t c$ (Bruneau et al., 1997), $d l x 2$ (Akimenko et al., 1994), $f k d 6$ (Kelsh et al., 2000), $f k d 7$ (Odenthal and NussleinVolhard, 1998), foxA2 (Strahle et al., 1993), hoxb2 (Yan et al., 1998), $\mathrm{Hu}$ (Marusich et al., 1994), $n k x 2.3$ (Lee et al., 1996), $n k x 2.5$ (Lee et al., 1996) and $r d r$ (Delot et al., 1999), Zn5 (Trevarrow et al., 1990). Cartilage was stained with Alcian Blue as described previously (Kimmel et al., 1998). To monitor cell death, we used either Acridine Orange at $5 \mu \mathrm{g} / \mathrm{ml}$ (Delot et al., 1999) or TUNEL analysis (Apoptag in situ kit, Intergen).

\section{RESULTS}

\section{Cas activity is essential for the development of the pharyngeal skeleton}

The pharyngeal skeleton of zebrafish is composed of a dorsal neurocranium and a ventral viscerocranium, which surrounds the pharyngeal cavity (Kimmel et al., 1998). The larval neurocranium is composed of a number of paired cartilage elements, including the median trabeculae, which fuse anteriorly into the ethmoid plate, and the parachordals 
Fig. 1. Chondrogenic neural crest cells do not form pharyngeal cartilage elements in endoderm mutants.

$(\mathrm{A}, \mathrm{C})$ (ventral views) Cartilages from 4-day-old embryos were stained with Alcian Blue, revealing the

viscerocranium (green labels) and the neurocranium (red labels).

Pharyngeal arch cartilages and trabeculae (arrowhead) are absent (B) or reduced (C) in cas and bon.

$(\mathrm{D}, \mathrm{E})$ Transverse sections as indicated by dashed lines in A and B. Stars indicate the heart/hemicardia and arrowheads indicate arch cartilages. $(\mathrm{F}, \mathrm{G})$ radar expression in $48 \mathrm{hpf}$ embryos (lateral view).

(H-O) Expression of the marker $d l x 2$. (H-M) Dorsal views. (H,I) Formation of the three streams I, II and III of $d l x 2$-positive neural crest cells is initially normal in cas embryos. $(\mathrm{J}, \mathrm{K})$ At $24 \mathrm{hpf}$, stream III, which is already segmented in wild type, is no longer detectable in mutants. (L,M) At 30 hpf, expression of $d l x 2$ in cas embryos is limited to stream I. $(\mathrm{N}, \mathrm{O})$ Lateral views. At $48 \mathrm{hpf}$, expression of $d l \times 2$ in cas embryos is restricted to a thin line posterior to the eye. (P-S) At $24 \mathrm{hpf}$, expression of $f k d 6(\mathrm{P}, \mathrm{Q})$ as well as $\mathrm{Hu}(\mathrm{R}, \mathrm{S})$ appears unaffected in cas. b, branchial arches; e, eye; ep, ethmoid plate; f, fin; h, hyoid; m, mandibular; mo, mouth; no, notochord; ov, otic vesicle; pc, parachordal; t, trabeculae.
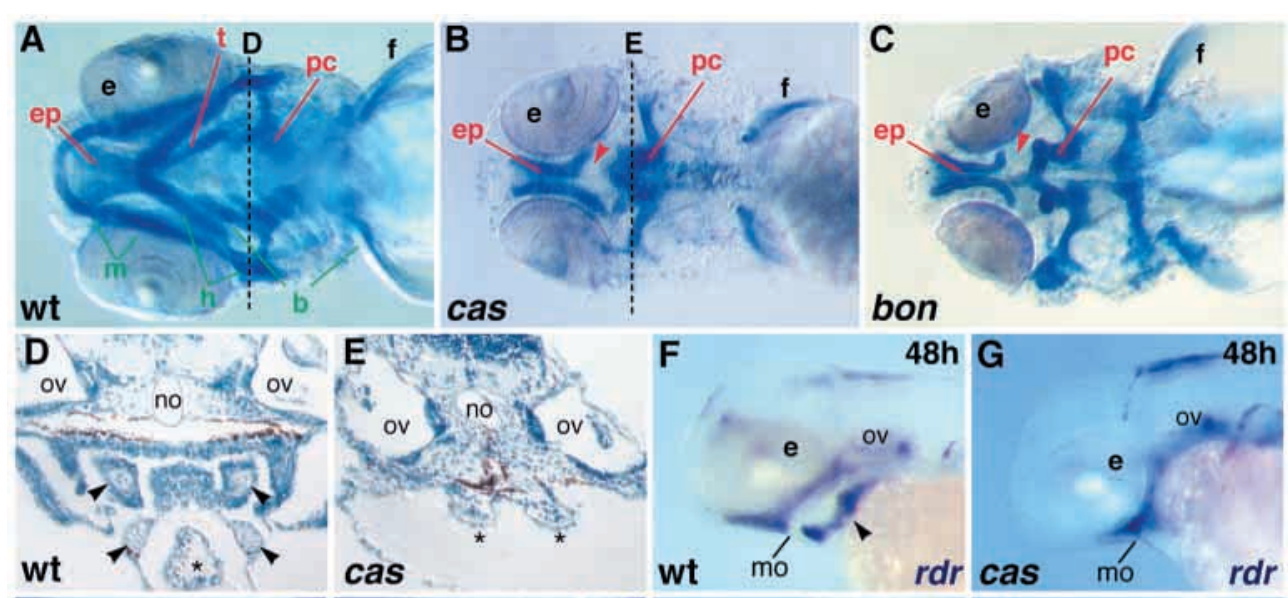

$48 \mathrm{~h} \mathrm{G}$

$48 h$
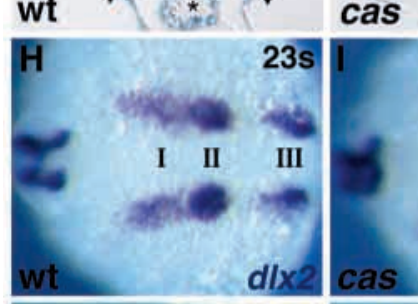

wt
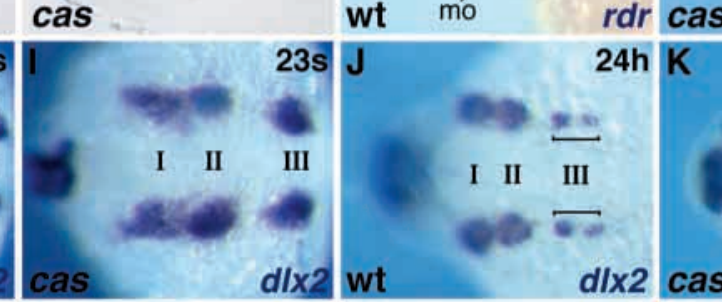

$24 \mathrm{~h}$ K

rdr

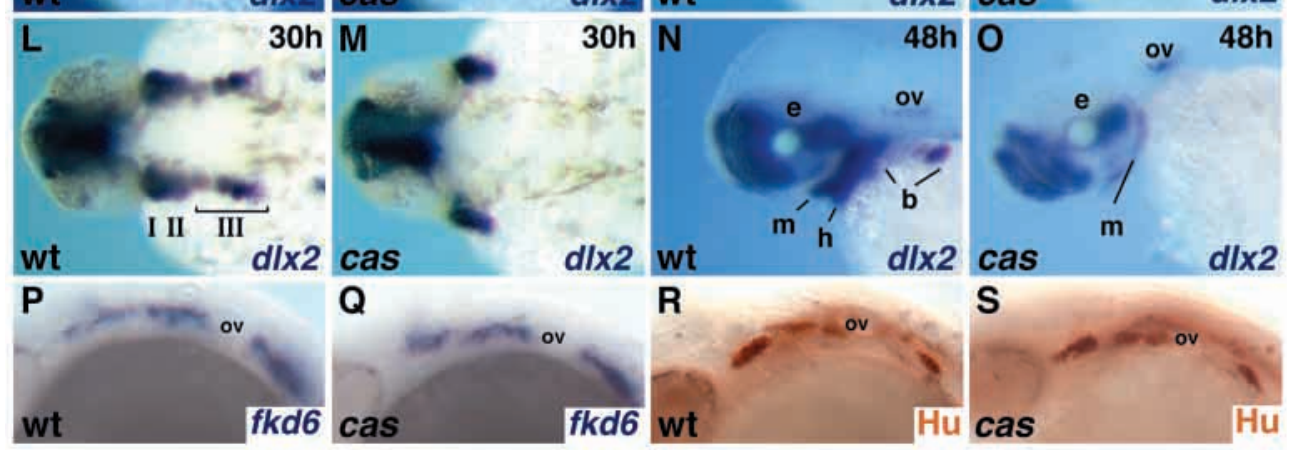

posteriorly. The larval viscerocranium consists of a series of seven pharyngeal arches composed of a repeated pattern of cartilages (Schilling, 1997). The two most anterior arches are the mandibular and the hyoid, which form the jaw and its support. Posteriorly, there are five smaller branchial arches that support the gills. Pharyngeal endoderm has been shown to influence the later stages of differentiation of mandibular neural crest cells into chondrocytes in amphibians (Seufert and Hall, 1990), but whether endoderm is more generally required for controlling the fate of chondrogenic cranial neural crest cells and when it acts is not clear. Thus we examined cartilage elements in mutants known to be defective in endoderm, bonnie and clyde (bon) and casanova (cas). Cartilage can be easily visualised by Alcian Blue staining of proteoglycans at 4-5 days. As compared to wild-type siblings, cas mutant larvae lack the entire viscerocranium (Fig. 1A,B) while the neurocranium is only slightly reduced, with the exception of the trabeculae which are reduced or absent and fail to fuse with the more posterior parachordals in $64 \%$ of mutants $(n=114$; arrowhead in Fig. 1B). The absence of cartilage was confirmed by the analysis of sectioned embryos (Fig. 1D,E). Similarly, bon embryos lack most or all of the viscerocranium (Fig. 1C). Therefore, consistent with a requirement for endoderm in the formation of the viscerocranium, both cas and bon mutants lack pharyngeal cartilage.
To determine the stage at which the process of cranial cartilage formation is interrupted in cas mutants, we analysed the expression of molecular markers of cartilage precursors. Differentiated cartilage is first detected at 54 hours post fertilisation (hpf) (Schilling and Kimmel, 1997). In 48 hpf wild-type embryos, the BMP-related gene radar $(r d r)$ is expressed in mesenchyme in the position where the anterior neurocranium will form, dorsal to the developing mouth, and viscerocranium ventral to the mouth (arrowhead in Fig. 1F) (Delot et al., 1999). Only the dorsal, neurocranial domain of expression is present in cas mutants (Fig. 1G). Likewise, in wild type, neural crest cells that will form the arch skeleton express the homeobox transcription factor $d l x 2$ during their early migration and subsequent condensation (Fig. 1H,J,L,N) (Akimenko et al., 1994; Ellies et al., 1997). During midsomitogenesis, $d l x 2$ is detected as three bilateral streams of cells on the sides of the midbrain and hindbrain, which will populate respectively the first, second and remaining five pharyngeal arches. All three streams are present in cas mutants (Fig. 1I) indicating that neural crest cells form and migrate. In wild-type embryos, the most posterior $d l x 2$ positive stream of cells (stream III) then progressively splits into two, three, four and eventually five cell groups, each separated by endodermal pouches of the pharynx. In cas mutants, dlx 2 expression in these streams of neural crest is 
progressively lost from posterior to anterior (Fig. 1K,M), with the exception of a thin band of mandibular mesenchyme just posterior to the eye (Fig. 1O). A similar loss of dlx3 expression, which also labels chondrogenic neural crest was observed in cas homozygous mutants (data not shown). Thus, the absence of the pharyngeal arch cartilages in cas homozygous larvae probably results from an earlier neural crest defect.

To determine if the phenotype observed in cas results from a more general neural crest defect, we also analysed various other neural crest cells derivatives. Between 48-72 hours the number and distribution of melanocytes and mesenchyme migrating in the median fin folds are identical in cas homozygous embryos and their wild-type siblings (data not shown). Likewise, expression of fkd6, which labels premigratory cranial neural crest (12 hpf; data not shown) and later putative glial precursors at $24 \mathrm{hpf}$ (Kelsh et al., 2000), as well as $\mathrm{Hu}$ expression in cranial and trunk sensory neurons, also appear normal in cas homozygous embryos (Fig. 1P-S). Conversely, and consistent with cartilage defects, expression of collagen $2 a$ in the forming arches at $48 \mathrm{hpf}$ is absent in cas mutants (data not shown). Taken together, these results suggest that the cas mutation specifically affects a chondrogenic subset of neural crest in the head.

\section{Fate of chondrogenic neural crest cells in cas embryos}

The progressive loss of $d l x 2$ expression in cas mutants could result from a fusion of neural crest streams, local downregulation of gene expression or death of cells populating streams II and III. To distinguish between these possibilities, we followed cranial neural crest morphogenesis in cas mutants. Morphological inspection with Nomarski optics at $24 \mathrm{hpf}$ revealed two abnormally large, bilateral arch primordia flanking the head, one anterior (Fig. 2A,B) and one posterior to the otic vesicle in the positions of streams II and III. At higher magnification, cells in the anterior primordium appeared disorganised unlike wild-type embryos in which neural crest cells align in vertical rows (not shown). We also observed an accumulation of mesenchymal cells in the position of stream I, immediately posterior to the eye.

To examine the segmental origins of these abnormal arch
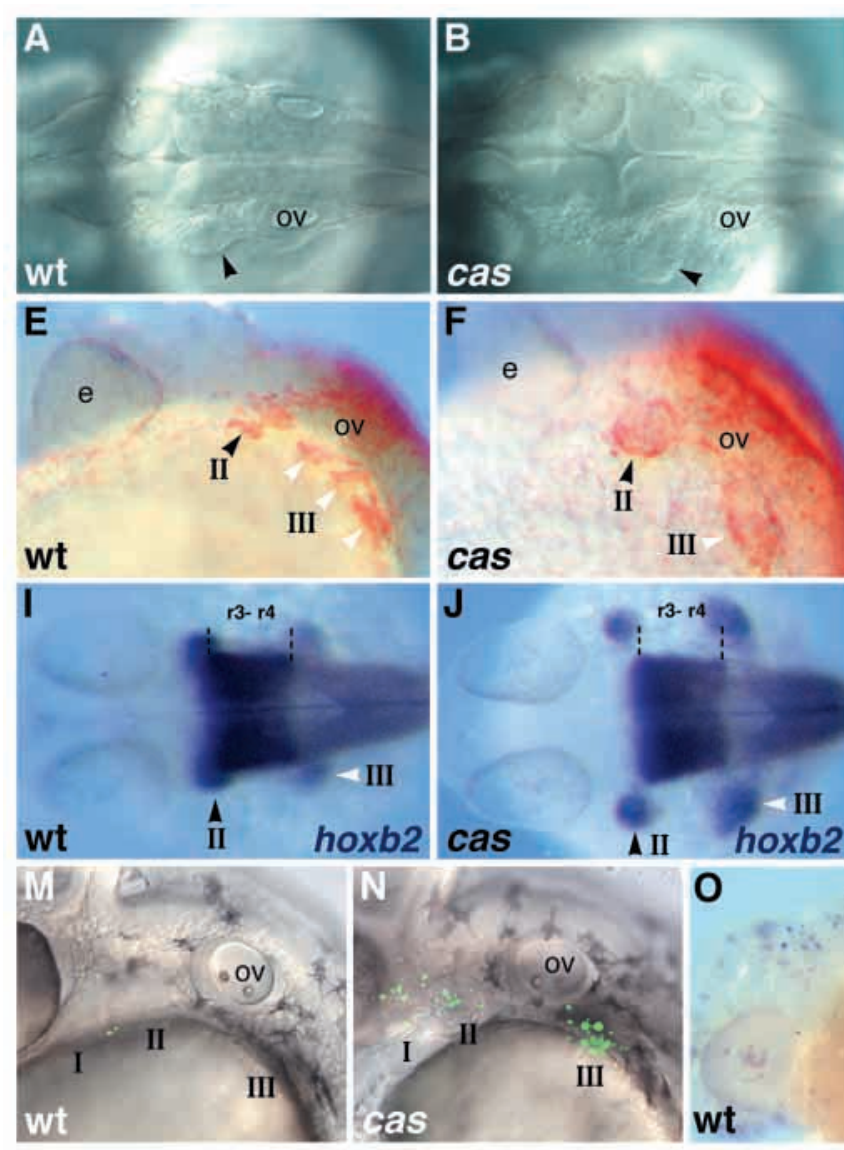
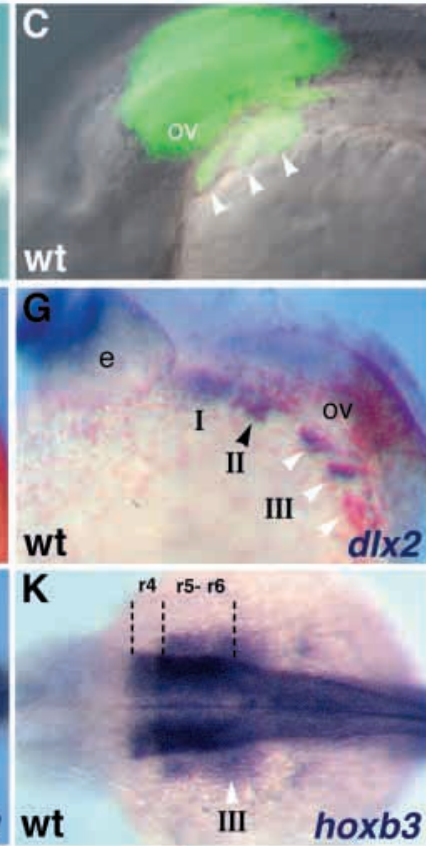

AII
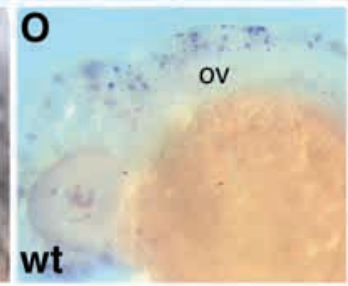
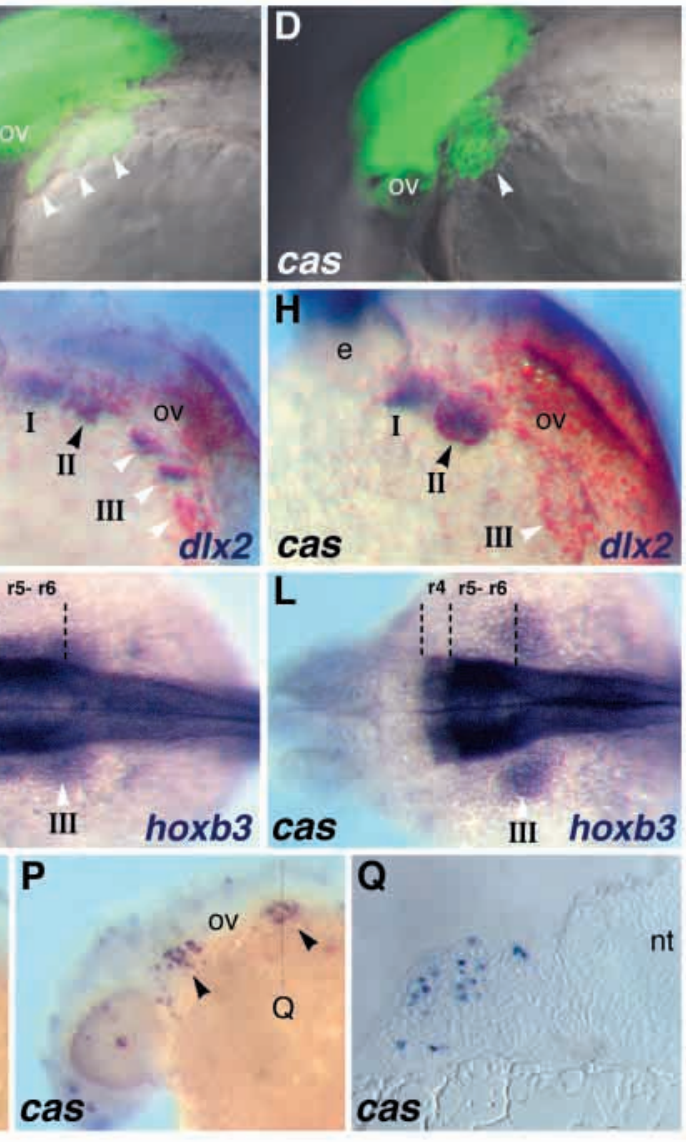

Fig. 2. Fate of chondrogenic neural crest cells in wild-type and cas embryos. (A,B) Dorsal view of the head of live 24 hpf embryos. Note in cas an additional lobular structure (arrowhead). (C,D) Dorsal neuroectoderm and neural crests posterior to the otic vesicle were labelled by activation of DMNB-caged fluorescein (see Materials and Methods). By $28 \mathrm{hpf}$, neural crest stream III (white arrowheads) has initiated segmentation in wild-type but not in cas embryos (D). (E-H). Same as in C,D but DMNB-caged fluorescein was activated in precursors of streams II (black arrowhead) and III. Embryos were fixed at $28 \mathrm{hpf}$ and processed for detection of fluorescein (E-H, orange) and $d l x 2$ (G,H, purple). (E-H) Streams I, II and III are present but dlx2 expression is downregulated in stream III in cas embryos (H). (I-L) Dorsal views. hoxb2 $(\mathrm{I}, \mathrm{J})$ and hoxb3 $(\mathrm{K}, \mathrm{L})$ expressions at $28 \mathrm{hpf}$ reveal the correct AP value of stream II and III, which adopt an ectopic lateral position in cas embryos. (M,N) Lateral view. $30 \mathrm{hpf}$ embryos were labelled with Acridine Orange, revealing increased cell death in the region of the three cranial neural crest streams in cas embryos. (O-Q) These results were confirmed by TUNEL analysis and sectioning (Q). e, eye; ov, otic vesicle. 
primordia, we followed labelled neural crest cells in cas mutants using the detailed fate map for premigratory cranial neural crest generated from $12 \mathrm{hpf}$ wild-type embryos (Schilling and Kimmel, 1994). The photoactivatable dye DMNB-caged fluorescein was injected into the progeny of cas heterozygotes at the one-cell stage and the dye was UVactivated in a local region of the cranial neuroepithelium when embryos reached the 5- to 7-somite stage. This technique typically labelled both neural tube and neural crest cells in the same experiment, conveniently marking migrating neural crest as well as their position of origin. In wild-type pharyngula stage (28 hours) embryos, fluorescent cells were later observed in particular arches depending on their original position. For instance, photoactivation in a region immediately posterior to the otic vesicle in wild type labels stream III and later the branchial arches, which by this stage are separated by endodermal pouches (Fig. 2C). Photoactivation at a similar AP position in cas mutant embryos resulted in labelled cells in the position of stream III and posterior arch primordium, but these were not subdivided into separate arches (Fig. 2D). Conversely, photoactivation in regions anterior to the otic vesicle labelled neural crest cells in streams I and II and the anterior arch primordia in both wild type and cas (data not shown). These results suggest that cas mutant neural crest cells migrate and roughly populate the appropriate migration streams along the AP axis and are specified correctly.

To confirm this, we photoactivated DMNB-caged fluorescein in larger stretches of crest at $12 \mathrm{hpf}$, encompassing territories both anterior and posterior to the otic vesicle and co-localized the lineage tracer (orange) (Fig. $2 \mathrm{E}-\mathrm{H})$, with the ectomesenchymal marker $d l \times 2$ at $28 \mathrm{hpf}$ (purple) (Fig. 2G,H). In contrast to wild-type embryos (Fig. $2 \mathrm{G}$ ), stream III is dlx2-negative in cas mutants (Fig. $2 \mathrm{H}$ ). Thus in cas, precursors of the branchial arches migrate ventrally, to reach a position over the yolk that is in register with their normal AP fate, but they lose dlx2 expression. At similar stages, stream III cells continue to express hoxb2 and hoxb3, another indication that they retain their correct AP positional value (Fig. 2IL). To determine if neural crest cells in these abnormal arch primordia in cas die, we analysed cell death patterns in wild-type and cas homozygous embryos using both Acridine Orange staining (Fig. 2M,N) and TUNEL analysis (Fig. 2O-Q). Strikingly, while little cell death is observed in arches between 24-48 hpf in wild type, significant cell death is observed in this region in cas homozygous embryos. This cell death is first detectable at $24 \mathrm{hpf}$ and becomes more pronounced by $30 \mathrm{hpf}$, occurring throughout the mediolateral extent of the arch (Fig. 2Q). Thus, at least some ectomesenchymal neural crest cells require cas function for maintaining their identity and survival.

\section{The requirement for cas in head cartilage formation is cell non-autonomous}

cas is a sox-related transcription factor required autonomously within endoderm cells for their development and differentiation. Although no expression of cas can be detected in neural crest cells (Dickmeis et al., 2001; Kikuchi et al., 2001), cartilage defects might reflect an autonomous requirement for cas/sox expressed at a very low level. To exclude this possibility, we used mosaic analysis to test the capacity of cas neural crest cells to differentiate into cartilage when transplanted into wild-type hosts. Chondrogenic cranial neural crest that forms the jaw (mandibular arch) has been mapped at $12 \mathrm{hpf}$ (Schilling and Kimmel, 1994) to the most dorsal tiers of premigratory neural crest cells in an oblong primordium located between the eye and the otic vesicle. FITC-dextran-labelled cranial neural crest cells were grafted from this location in cas mutants into the same chondrogenic region of wild-type host embryos and their fates were followed until day 3 (Fig. 3B). Most of these cas mutant cells differentiated into typical neural crest derivatives, notably contributing with high frequency to the cartilage elements of the mandibular and hyoid arches $(50 \%, n=12$; Fig. 3C,D). Controls in which wild-type cranial neural crest cells were transplanted into wild-type hosts formed cartilage at a similar frequency $(56 \%, n=16)$. In contrast, wild-type cells transplanted into cas homozygous embryos never differentiated into cartilage $(0 \%, n=15)$. These results demonstrate that cranial neural crest cells in cas have the ability to develop into cartilage when placed into a wild-type environment and thus cas activity is required nonautonomously. cas must be required in the premigratory or migratory environment of these neural crest cells to control their participation in pharyngeal cartilage formation. An attractive candidate for the source of such environmental influences is the endoderm because, (i) this tissue is absent in cas, (ii) neural crest cells in the arches contact pharyngeal endoderm prior to chondrogenesis (Schilling and Kimmel, 1994), and (iii) endoderm has been shown to influence the differentiation of chondrocytes in amphibians (Seufert and Hall, 1990). To test this idea, it was essential to restore endoderm in cas homozygous embryos and analyse its consequences for the formation of the viscerocranium.

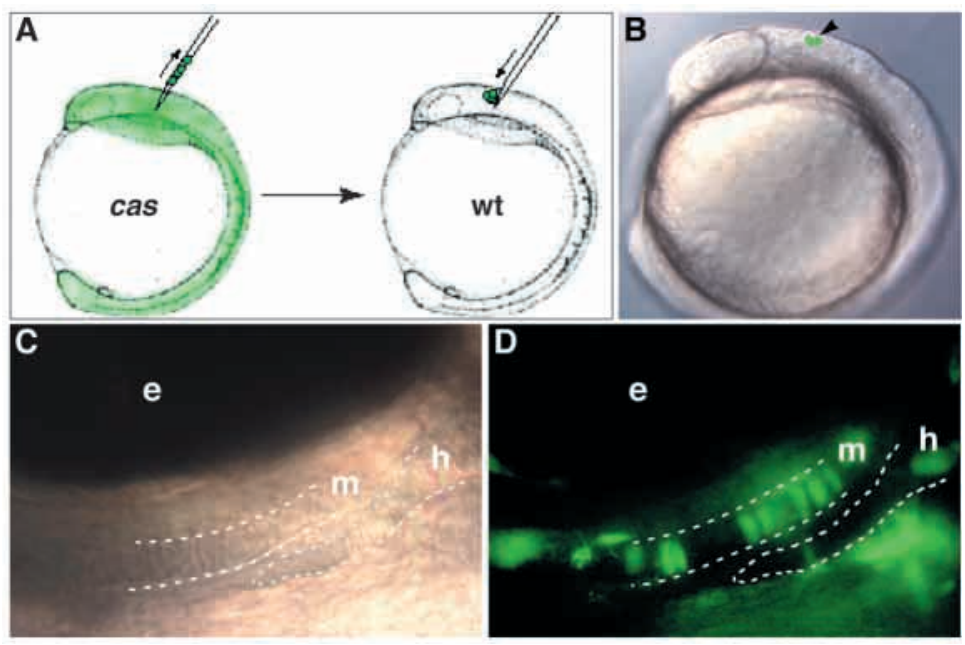

Fig. 3. cas neural crest cells differentiate into cartilage in wild-type embryos. (A) Diagram of the transplant procedure. (B) At the 10-somite stage, a few FITC-labelled (green) premigratory neural crest cells were transplanted from a cas embryo into a wild-type host. (C) At 3 days, in similarly transplanted embryo, mandibular and hyoid cartilage can be seen close to the eye. (D) cas neural crest have contributed to these cartilages in a characteristic stack-of-penny arrangement. e, eye; h, hyoid; m, mandibular. 


\section{Endoderm-mediated restoration of pharyngeal skeleton in cas}

cas acts downstream of nodal/tar signalling, thus activation of nodal/tar signalling (for example by injection of Tar*) will not rescue endoderm formation in cas homozygous mutant embryos (Alexander and Stainier, 1999; Dickmeis et al., 2001). Transplants of marginal cells at gastrulation in zebrafish, while effective for targeting the mesodermal layer, rarely contribute to the endoderm, making it very difficult to use such an approach to study the function of endoderm during development (Alexander et al., 1999; David and Rosa, 2001). However, activation of the Tar/Nodal signalling pathway is sufficient to autonomously specify wild-type cells as endoderm. tar*-activated cells transplanted, at the blastula stage, into the margin of either wild-type or more importantly cas embryos express endoderm markers at gastrulation and differentiate into classical endoderm derivatives, including pharyngeal endoderm (Fig. 4A-C) (Bally-Cuif et al., 2000; David and Rosa, 2001). This gave us a unique tool for testing the role of endoderm in pharyngeal development in cas.

We analysed cartilage in 4-day-old cas mutant larvae that had received grafts of small groups of control or tar*-activated wild-type cells. Grafting of control, untreated cells, although they populated head mesodermal structures, never restored endoderm (except for a few cells) or viscerocranial cartilage in cas mutants (Table 1). In contrast, grafting of tar*-injected wild-type cells into cas mutants efficiently restored endoderm and rescued neighbouring viscerocranial elements $(69 \%, n=36)$ (Fig. 4E-G; Table 1). Interestingly, in tar*-grafted cas homozygous mutants trabeculae were present that fused with the parachordals in $80 \%$ cases (as compared with $36 \%$ in controls), suggesting that grafting of endoderm also rescued elements of the neurocranium. To determine if tar*-grafted cells also rescued neural crest specification in arch primordia we compared the expression of dlx2 in 30-hour-old cas embryos with and without tar*-activated wild-type cells in the pharynx. No rescue was observed when grafted cells were located in any part of the endoderm outside the pharynx. Quite strikingly, however, tar*-injected cells in the pharyngeal region of cas hosts restored $d l x 2$ expression in neural crest streams, frequently leading to an almost normal $d l x 2$ expression pattern on the side of the embryo harbouring the grafted cells (Fig. $4 \mathrm{H})$. Analysis of sections further revealed that grafted cells were always adjacent to the rescued $d l x 2$ domains but never expressed $d l x 2$ themselves (Fig. 4I). We conclude that introduction of endoderm into cas homozygous embryos rescues the normal developmental pathway for chondrogenic neural crest cells in a non-autonomous fashion, possibly through signals from the endoderm-derived pharyngeal pouches. This strongly indicates a requirement for endoderm as a key component in the control of neural crest fate and viscerocranium formation.

\section{FGF signalling is required to control the fate of chondrogenic neural crest cells and their differentiation/morphogenesis}

Pharyngeal arch formation thus critically requires the interaction between migrating mesenchymal neural crest cells and the endodermal epithelium of the pharyngeal pouches to allow proper neural crest cells fate, but the molecular nature of the signals provided by the endoderm remains to be defined. Epithelial-mesenchymal interactions, particularly based on studies on the model of limb and lung formation are often mediated by extracellular secreted factors, including members
Fig. 4. Restoration of endoderm rescues arch cartilage formation in cas embryos. (A) At blastula stage, wild-type cells expressing Tar* were transplanted to a cas host. (B) Side view of the head region at $30 \mathrm{hpf}$. Grafted cells (green) have differentiated into endodermal derivatives, here pharyngeal endoderm. The arrow indicates a forming pouch. (C) These pouches restored by grafted cells (black nuclei) were stained with the Zn5 antibody (brown). (D,E) At 4 days, grafted cas embryos (E) but not controls (D) develop arch cartilage elements revealed by Alcian Blue staining. Anterior to the left.

(F) Horizontal section of a grafted cas embryo showing the differentiation of cartilage elements (blue) lined by endodermal pouches derived from transplanted cells (brown). Anterior to the top. (G) Close-up of the region containing grafted cells and rescued cartilage. (H,I) 30 hpf grafted cas embryos rescues $d l x 2$ expression in
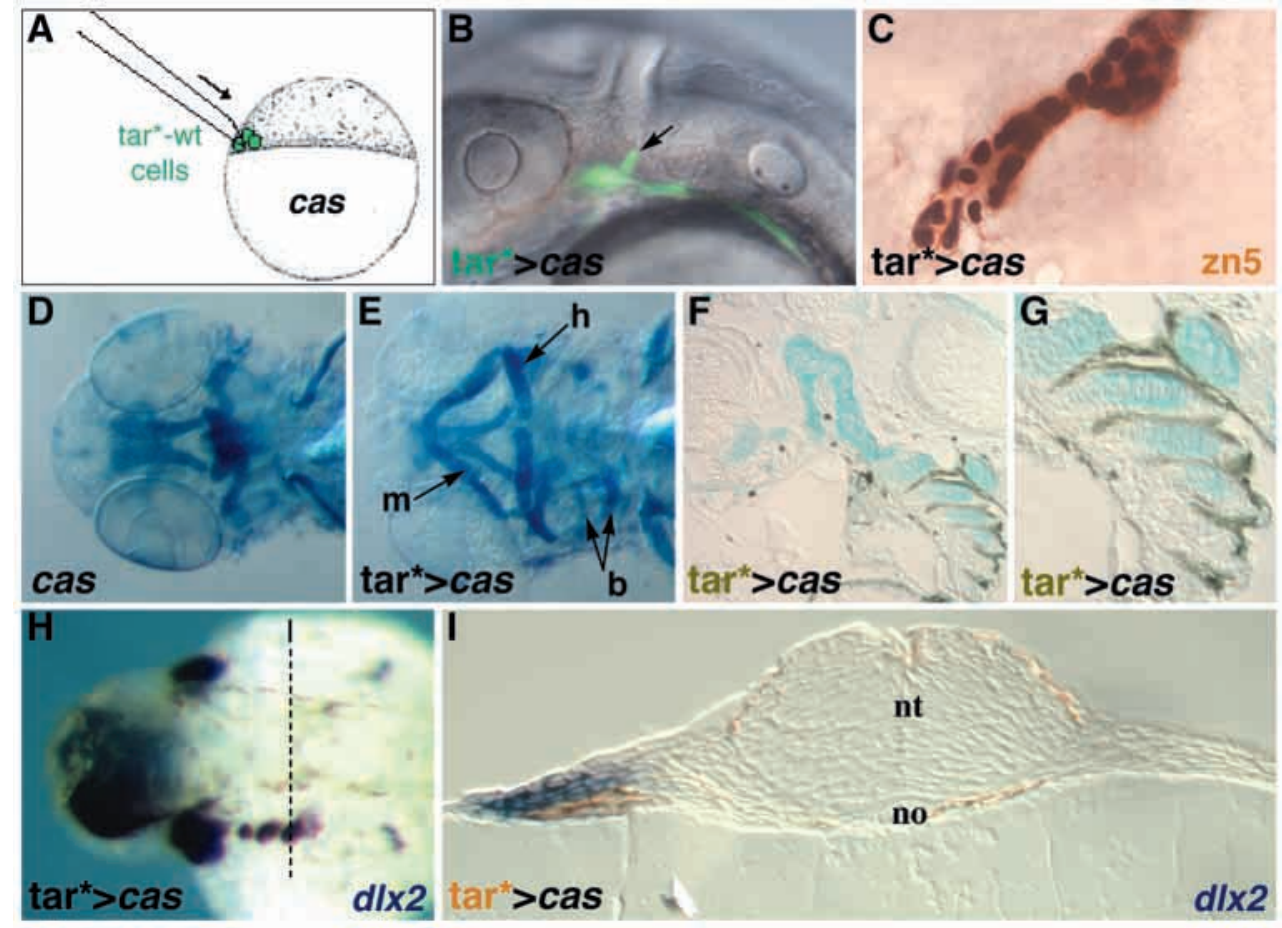

posterior cranial neural crest cells (H, dorsal view, anterior left). (I) Cross section (indicated in $\mathrm{H}$ ) showing that wild-type transplanted cells (brown) abut the cluster of $d l x 2$-positive neural crest cells (blue). Note the absence of $d l x 2$ expression on the control ungrafted side of the embryo. Dorsal to the top. b, branchial arch; h, hyoid; m, mandibular; no, notochord; nt, neural tube. 
Table 1. Pharyngeal arch cartilage formation requires $f g f 3$

\begin{tabular}{|c|c|c|c|c|c|}
\hline & & $\begin{array}{c}\text { No } \\
\text { arches }\end{array}$ & $\begin{array}{l}\text { Anterior } \\
\text { arches }\end{array}$ & $\begin{array}{c}\text { Branchial } \\
\text { arches } 1 \text { to } 4\end{array}$ & $\begin{array}{c}\text { Branchial } \\
\operatorname{arch} 5\end{array}$ \\
\hline Su5402 treatment of wt embryos & $n=50$ & $42 \%$ & $58 \% *$ & $2 \%$ & $10 \%$ \\
\hline Fgf3-Morpholino injection at the one cell stage into wt embryos & $n=60$ & $17 \%$ & $83 \%$ & $8 \%$ & $53 \%$ \\
\hline Transplant into cas of wt cells injected with GFP & $n=26$ & $100 \%$ & $0 \%$ & $0 \%$ & $0 \%$ \\
\hline Transplant into cas of wt cells injected with tar* & $n=36$ & $31 \%$ & $69 \%$ & $36 \%$ & n.d. \\
\hline Transplant into cas of wt cells injected with tar* and fgf3-Mo & $n=18$ & $44 \%$ & $56 \%$ & $0 \%$ & $0 \%$ \\
\hline \multicolumn{6}{|l|}{$\begin{array}{l}\text { Numbers represent the percentage of embryos in a treatment group. } \\
\text { *Extremely reduced, see Fig. 6C. }\end{array}$} \\
\hline
\end{tabular}

of the Hedgehog, bone morphogenetic protein (BMP) and fibroblast growth factor (FGF) families (Tickle and Munsterberg, 2001). We have tested the potential function of FGFs in pharyngeal cartilage formation. We first blocked FGF signalling starting around the stage at which neural crest cells first encounter endoderm pouches. Strikingly, midsomitogenesis embryos (15-somite stage) incubated for 24 hours in a solution of the synthetic FGF inhibitor SU5402 (Mohammadi et al., 1997; Shinya et al., 2001) did not develop any viscerocranial cartilage at 4 days except for a few small nodules in the positions of the mandibular and hyoid, and a severely reduced neurocranium (Fig. 6B,C and Table 1). Furthermore, similar to cas mutants, the expression of $d l x 2$ in neural crest-derived mesenchyme was largely downregulated at $48 \mathrm{hpf}$ in SU5402-treated embryos, and extensive cell death was observed in the region of chondrogenic crest cells at $36 \mathrm{hpf}$ (data not shown). This indicates that FGF signalling is crucial for the fate of chondrogenic neural crest at the time they migrate between the pharyngeal pouches.

$f g f 8$ may be responsible for some of this activity, as $f g f 8$ zebrafish mutants or morphants (Roehl and NussleinVolhard, 2001) (N. B. D. and F. M. R., data not shown) display a variable reduction in arch cartilage. However $f g f 8$ itself does not appear to be expressed in the pharyngeal endoderm (Roehl and Nusslein-Volhard, 2001) (N. B. D. and F. M. R., data not shown). $f g f 3$ may be involved in arch formation/patterning since it is expressed in the pharyngeal endoderm in zebrafish during stages of neural crest migration and differentiation (Fig. 5) (Kudoh et al., 2001; Furthauer et al., 2001). At the 12-somite stage, in addition to its expression at the mid-hindbrain boundary and rhombomere 4, $f g f 3$ expression is detectable in the pharyngeal endoderm region extending along both sides of the embryo alongside rhombomeres 1-3 (Fig. 5A,D,J). During later somitogenesis, endodermal expression of $f g f 3$ becomes restricted to three bilateral stripes corresponding to the already formed pharyngeal pouches. Expression in the most anterior site is transient and corresponds to the first endodermal pouch between arches I and II. At $24 \mathrm{hpf}, f g f 3$ expression wears off anteriorly but is maintained in two more posterior pouches. At this stage, the two walls of each pharyngeal pouch are juxtaposed and appear as a double layer epithelium, each facing a stream of $d l x 2$-positive neural crest cells. Double staining for $d l x 2$ and $f g f 3$ confirmed that $f g f 3$ is expressed in endodermal pouches and further shows that its expression is restricted to the posterior half of the pouch (Fig. 5K). Later on and up to $48 \mathrm{hpf}$, expression of $f g f 3$ is maintained in posterior pouches (Fig. $5 \mathrm{G}, \mathrm{H}$ and data not shown).

Consistent with the lack of endoderm in cas embryos, the endodermal expression of $f g f 3$ is absent in the mutant (Fig. 5I). The arch phenotype observed in cas could thus be due, at least in part, to this lack of $f g f 3$ expression. To test this possibility, we injected a morpholino directed against $f g f 3$ or against $f g f 4$, as a control, into wild-type embryos. Strikingly, $f g f 3$ morphants displayed a frequent loss of branchial arches (except the most posterior, arch 7) and a hyoid arch with inverted AP polarity

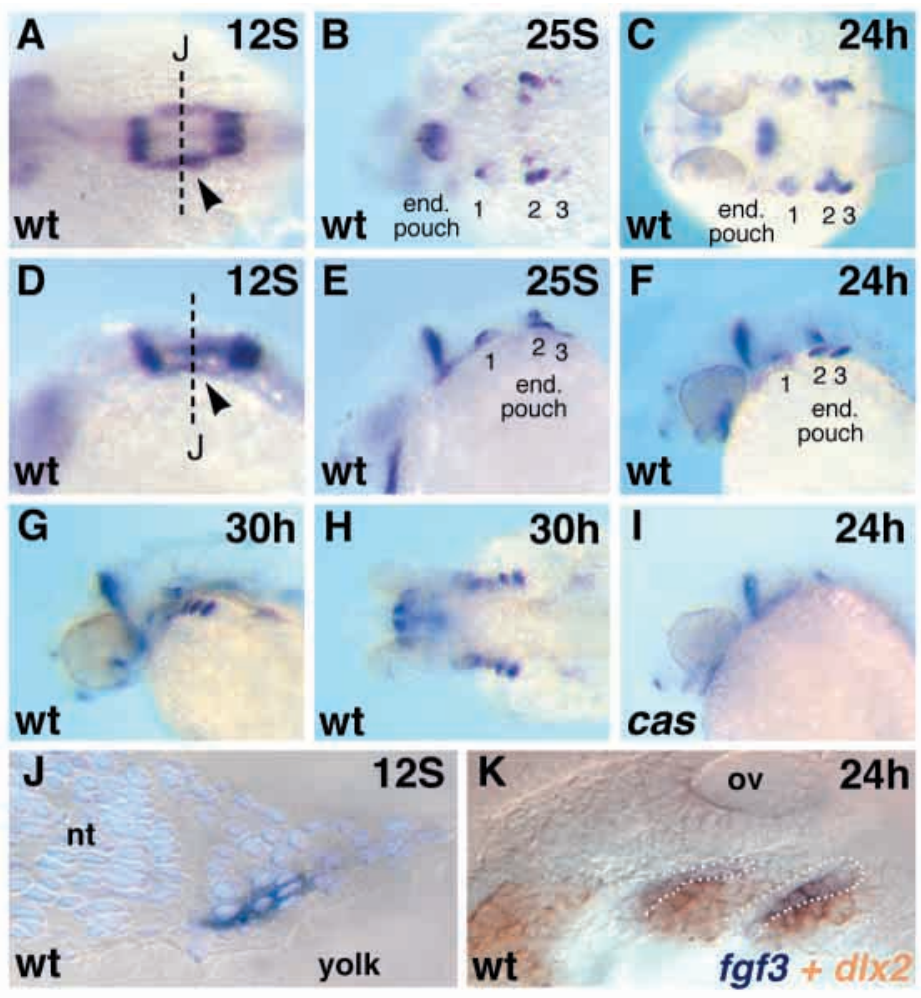

Fig. 5. Expression of $f g f 3$ in pharyngeal pouches. Dorsal (A-C) and lateral (D-G) views of embryos stained for $f g f 3$ expression. Endodermal expression is indicated on one side (arrowheads and labelling). Anterior to the left. (I) The endodermal expression of $f g f 3$ is absent in cas embryos. (J) Section of a 12-somite embryo showing expression of $f g f 3$ in flat cell abbutting the yolk sack, thereby adopting the normal position and shape of endoderm cells. (K) Enlarged lateral view from the otic region. 24 hour-old embryos were double stained for $f g f 3$ (blue) and $d l x 2$ (red). $f g f 3$ is expressed in the posterior part of posterior pharyngeal pouches (white dots) and abuts $d l x 2$-expressing neural crest cells. ov, otic vesicle. 


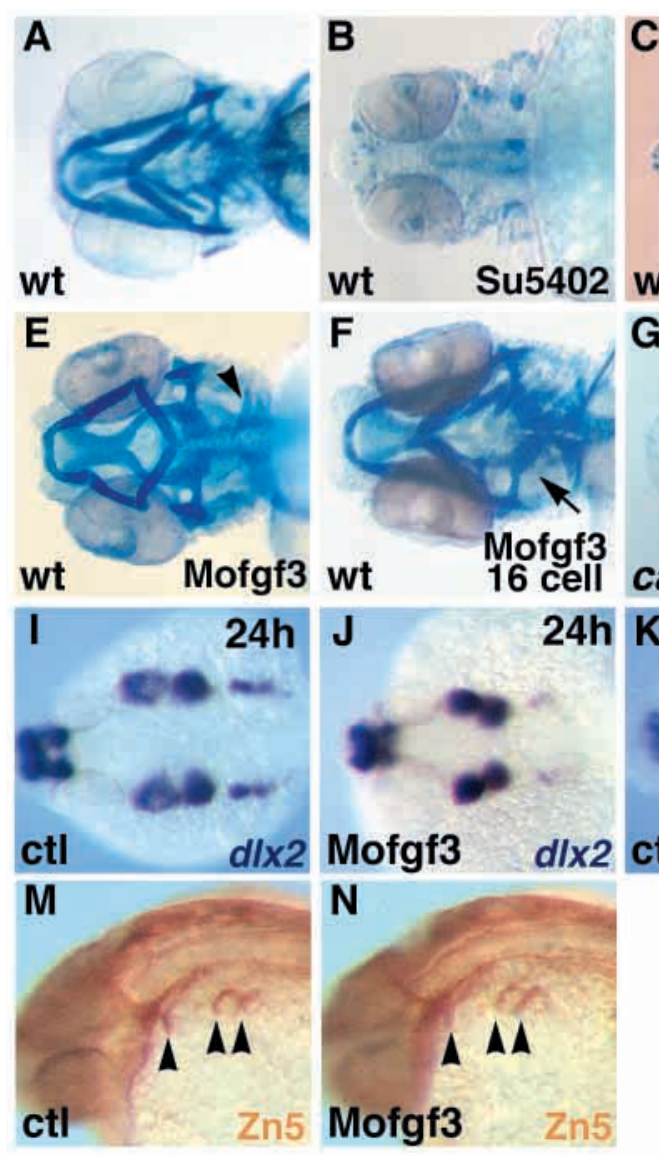

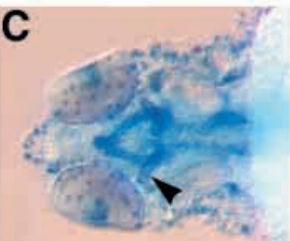
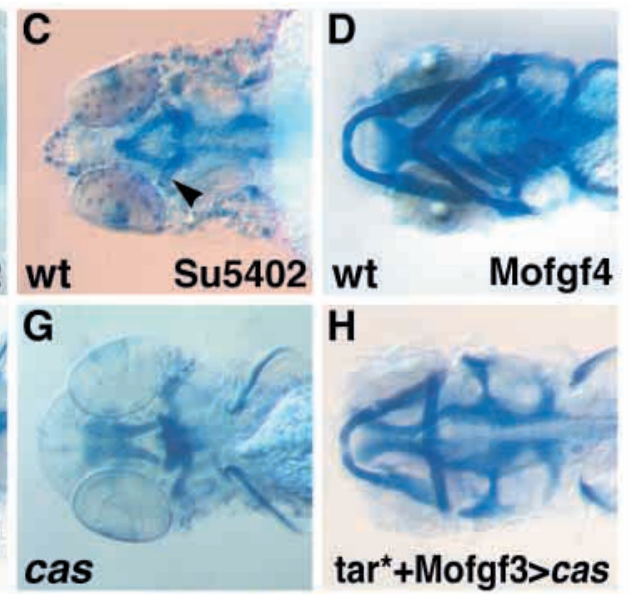

tar ${ }^{*}+$ Mofgf $3>$ cas
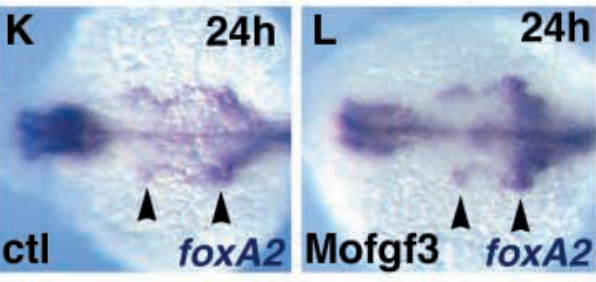

Fig. 6. $f g f 3$ is required in endoderm for the formation of posterior arches.

(A-H) Alcian Blue staining of 4-day-old embryos. Ventral views, anterior to the left. (A-F) Wild-type embryos were either left untreated (A), immersed in Su5402 $(\mathrm{B}, \mathrm{C})$ or injected with morpholinos as indicated in lower right corner (D-F). (B) Su5402 prevents head cartilage formation, except for some very reduced anterior elements (arrowhead in C).

$(\mathrm{D}, \mathrm{E})$ Cartilage in $f g f 4$ morphants appears normal but $f g f 3$ morphants do not develop branchial cartilage (except the fifth one, arrowhead), and develop a hyoid cartilage with inverted AP polarity. (F) Localisation of $f g f 3$ morpholinos to anterior mesendodermal tissues is sufficient to disrupt branchial cartilage formation. The arrow indicates the injected side of the embryo. (G,H) Wild-type cells expressing Tar* and $f g f 3$ morpholinos were grafted to cas embryos as in Fig. 4. At 4 days, compared to control cas embryos $(\mathrm{G})$,

grafted embryos developed mandibular and hyoid arch cartilages but not branchial cartilages. (I-N) Dorsal and lateral views, anterior to the left. Embryos were either injected with $f g f 3$ morpholino or left untreated. In $f g f 3$ morphants, cranial neural crest of stream III downregulates $d l x 2$ expression (I,J). However pharyngeal endoderm differentiates normally as revealed by the expression of foxA2 (K,L) and the staining of pharyngeal pouches with Zn5 antibody $(\mathrm{M}, \mathrm{N})$.

(Fig. 6E and Table 1) similar to the phenotypes of many other known posterior arch mutants in zebrafish (Schilling et al., 1996b). Examination of $d l x 2$ expression in $f g f 3$ morphants showed that cranial crest streams form normally from the dorsal head region during somitogenesis (data not shown) but that $d l x 2$ expression is down-regulated at $24 \mathrm{hpf}$ in the most posterior stream (Fig. 6I,J). A simple explanation for these phenomena is that the $f g f 3$ morpholino interferes with pharyngeal endoderm development. However, pharyngeal endodermal markers such as axl/foxA2 or zn5 (Fig. 6K-N) appear to be unperturbed, making this explanation unlikely. Thus $f g f 3$ function is critically required for posterior cranial neural crest cells to maintain $d l x 2$ expression once they have reached the pharyngeal pouches and to develop a pharyngeal cartilage fate.

\section{fgf3 is required within the endoderm to control the fate of posterior cranial neural crest}

Expression of $f g f 3$ in the neural tube and/or in the pharyngeal endoderm could be responsible for the phenotypes of $f g f 3$ morphants. To determine which of these two sites of expression is essential for the control of chondrogenic crest fate, we performed two series of experiments. We first created chimaeric $f g f 3$ morphants by injecting the morpholino into one marginal cell of a 16-cell-stage embryo. Using this procedure, progeny of the injected cells populate mostly mesoderm- and endoderm-derived tissue (Peyrieras et al., 1998). Such a local knock-down led to asymmetric embryos lacking posterior arches only on the morpholino-injected side and this phenotype was strictly correlated with the localisation of morpholinopositive cells in the pharyngeal pouch region (Fig. 6F). These results indicate that $f g f 3$ activity is required within pharyngeal endoderm/mesoderm for the formation of branchial arches 1 to 4. To confirm this and to determine whether $f g f 3$ expression is required in mesoderm or more likely in endoderm, we analysed the restoration of pharyngeal arch formation in cas embryos by transplantation of tar*-induced endoderm from $f g f 3$ morphants. At 4 days, this led to an efficient restoration of anterior arches (mandibular and hyoid) but never rescued branchial arches, demonstrating that $f g f 3$ expression is required in the endoderm for formation of posterior arches (Fig. 6H and Table 1).

Altogether, our results show that $f g f 3$ expression in pharyngeal pouches is crucial for the control of posterior chondrogenic neural crest fate and pharyngeal arch formation. Interestingly, they also suggest that even if endoderm is necessary for the formation of all arches, this must be mediated by other signals in addition to FGFs in the anterior arches.

\section{DISCUSSION}

\section{Endoderm is essential for pharyngeal arch formation}

We provide evidence that endoderm is critical for the proper development of pharyngeal arch cartilages and the control of cranial neural crest fate. Reduction or absence of endoderm in bon and cas mutants correlates with reductions in all arch cartilages. However, defects in blood circulation at $24 \mathrm{hpf}$ and 
heart edema developing at later stages in these mutants, could indirectly lead to the later reduction of pharyngeal cartilage (Chen et al., 1996). Two results suggest that this is not the cause: (1) miles apart, another mutation causing circulation defects similar to cas and bon (Chen et al., 1996) does not disrupt cartilage (data not shown), and (2) rescue of endoderm and cartilage in cas occurs without rescuing circulatory defects or edema. In addition, we show that premigratory cas neural crest cells can form cartilage when transplanted among wildtype neural crest cells, demonstrating that cas function is not required in cartilage precursors but rather in their environment. Consistent with this result, cas is not expressed in neural crest cells or in their derivatives (Dickmeis et al., 2001; Kikuchi et al., 2001). Grafts of wild-type cells into the head mesoderm in cas do not restore arch cartilages suggesting that the skeletal defects are unlikely to result from mesodermal defects. In contrast, reintroduction of wild-type endoderm, by grafting Nodal/tar*-injected cells (David and Rosa, 2001) rescues cartilage formation of all pharyngeal arches in cas, arguing that endoderm is sufficient to induce, or at least allow, arch cartilage development.

Previous reports had suggested that endoderm is important in pharyngeal cartilage patterning. For instance, pharyngeal skeletal defects throughout the arches in the van gogh mutant in zebrafish correlate with defects in pharyngeal endoderm (Piotrowski and Nusslein-Volhard, 2000). Similarly, the use of a pan-RAR antagonist in mice led to defects in specification of arches 3 and 4 that the authors attributed to a disruption of signalling from the endoderm associated with these segments (Wendling et al., 2000). However, in the absence of mosaic analysis in both cases, the causal relationship between endodermal and skeletal defects remained speculative. Recent mosaic analyses in the chick have, however, provided stronger evidence that the endoderm provides AP positional information to the developing skeleton (Couly et al., 2002). In the present work, by means of genetic ablation and reintroduction of endoderm, we provide direct evidence for a crucial function of endoderm in the control of pharyngeal arch cartilage development. Whether or not endoderm acts directly on neural crest cells or requires an intermediate signal via neighbouring tissues cannot be ascertained at this point. However, the close spatial proximity between neural crest cells and endoderm within an arch (Trainor and Tam, 1995; Miller et al., 2000) as well as the coincidence between the early defects in neural crest-specific gene expression in cas mutants at stages when these cells first contact pharyngeal pouches (Schilling and Kimmel, 1994), argue in favour of a direct signal.

\section{Endoderm controls the fates of chondrogenic crest cells}

Until now, it was not clear whether endoderm had only a late function in controlling cartilage differentiation or acted at an early stage for correct migration of neural crest cells, for the maintenance of their identity, and/or for their survival. Our results suggest a role for endoderm in several of these early steps.

\section{Endoderm is required for midline convergence of cranial neural crest cells}

Arch precursor/neural crest cells normally stream from their origins adjacent to the hindbrain and migrate ventrally to surround the pharynx. In zebrafish initial streams form normally in the absence of endoderm, as shown by the expression of $d l x 2$ in cas mutants, suggesting that this tissue does not provide cues essential for initial migration. However, crest cells then accumulate in disorganized masses in cas mutants, and fail to migrate further ventrally or medially. Restoration of endoderm in cas mutants by transplantation results in the proper positioning of arch cartilage elements, strongly suggesting that these later aspects of crest migration are also restored. Thus endoderm appears important for convergence of cartilage precursors below the head. One possibility is that midline pharyngeal endoderm provides attractive signals for arch cartilages/neural crest precursors. Alternatively, endoderm may serve as a substrate for the migration of neural crest cells, and/or may passively displace adherent neural crest cells in the course of its own morphogenetic movement towards the midline (Warga and Nusslein-Volhard, 1999). Several bilateral organ anlage including, arch cartilage precursors, the precursors of the heart, blood vessels, kidneys and blood fail to converge or they exhibit delayed convergence toward the midline in endoderm deficient mutants such as cas and oep (Alexander et al., 1999; Peyrieras et al., 1998). Convergence of heart precursors can also be rescued by restoring endoderm, therefore strongly supporting the notion that endoderm has a key function in the control of convergence movements of many tissues toward the midline, a process essential for their morphogenesis (David and Rosa, 2001; Peyrieras et al., 1998).

\section{Endoderm is required for the maintenance of chondrogenic crest cells}

Soon after they have reached the yolk surface, neural crest cells in cas mutants downregulate $d l x 2$ and $d l x 3$ expression suggesting that endoderm is required to maintain expression in these cells. In support of this interpretation, mouse mandibular arch mesenchyme cultured by itself initially requires signals released from the adjacent oral epithelium to maintain the expression of crest-specific markers, though this later becomes epithelium-independent (Ferguson et al., 2000). With the markers available, we find no evidence that, in the absence of endoderm in cas, crest cells change their fates from chondrogenic to non-chondrogenic crest fates. Rather, by 30 hpf, crest cells in the arches undergo a period of extensive cell death, which may explain the lack of pharyngeal arch cartilage in the larva. Death may occur as a result of a failure in chondrogenic specification leading to the subsequent death of this subpopulation (fate mapping evidence suggests cartilage precursors are specified as early as premigratory stages) (Schilling and Kimmel, 1994) or, alternatively, cas and/or endoderm may have a more general requirement for crest cell survival in arches, including those cells that would form cartilage.

\section{Endoderm and the process of segmentation}

Chondrogenic neural crest cells migrate in distinct streams to generate distinct segmental structures in each arch (reviewed by Trainor and Krumlauf, 2001). Segmental migration has been proposed to reflect the segmental origins of crest cells in the hindbrain which then confers segmental patterning on the pharyngeal region (Noden, 1983). However, recent evidence from neural crest ablation studies in chick have demonstrated 
that segmentation of the pharyngeal endoderm does not depend on neural crest (Veitch et al., 1999). Likewise, our studies suggest that initial segmental migration of neural crest does not require cues from the endoderm. We use cell tracing studies to show that in the absence of endoderm in cas mutants, neural crest in the primordia of the mandibular and hyoid arches do not fuse and remain separate. Strict segmental lineage restrictions in zebrafish are already established at the onset of migration for these arches and probably result, at least in part, from factors intrinsic to the neural crest (Schilling and Kimmel, 1994). Endodermal morphogenesis may have some influence on the patterning of cranial crest once it reaches the arches, as suggested by recent analysis of the van gogh mutant (Piotrowski and Nusslein-Volhard, 2000). In support of this idea, we found that posterior neural crest cells, which normally split to form the five branchial arches, remain as a cluster in cas mutants suggesting that endodermal pouches are required to subdivide the branchial arches.

\section{Molecular nature of the endodermal signal}

One further challenge is to identify signals provided by the endoderm to neural crest cells. Several pieces of evidence support a major function of FGF signalling in the control of cranial neural crest fate and pharyngeal cartilage development. First, several FGFs and their receptors are expressed in the pharyngeal region (Launay et al., 1994; Mahmood et al., 1995; Wall and Hogan, 1995; Shinya et al., 2001; Kudoh et al., 2001; Furthauer et al., 2001). Second, FGF signalling is active in this region in amphibians following neural crest cell migration (Christen and Slack, 1999) and required to some extent for arch patterning in zebrafish and in mice (Roehl and Nusslein Volhard, 2001; Trumpp et al., 1999). We show that general inhibition of FGF signalling by Su5402 during arch morphogenesis prevents arch cartilage formation. Su5402 treatments also cause earlier downregulation of $d l \times 2$ and marked neural crest cell death, a situation strikingly reminiscent of the phenotypes observed in endoderm-deficient mutants.

Our results also implicate $f g f 3$ as an important regulator of cartilage development. Indeed, general disruption of $f g f 3$ function in $f g f 3$ morphants inhibits formation of posterior arches (though it has little effect on the most posterior, $5^{\text {th }}$ branchial arch, which is often unaffected in arch mutants) (Schilling et al., 1996b). However, $f g f 3$ is expressed both in the anterior neuroectoderm, from which cranial neural crest originates and in pharyngeal endoderm (Furthauer et al., 2001) (this work). To further define which of these sites is important for arch formation, we reduced $f g f 3$ function locally by creating chimaeric morphants. Consistent with a requirement for $f g f 3$ signals from the endoderm and not ectoderm, embryos harbouring $f g f 3$ morpholinos specifically in the cranial mesendoderm also exhibit a reduction or absence of branchial arch cartilages. In addition, cas mutants lack $f g f 3$ expression in the endoderm but not neuroectoderm. Altogether, these results suggest that $f g f 3$ is an essential signal from pharyngeal endoderm that induces skeletogenesis from the cranial neural crest.

Intriguingly, whereas endoderm and FGF signalling appear to be required for the whole set of arch cartilages, loss of $f g f 3$ function in morphants specifically prevents the formation of the posterior (gill-bearing) but not the anterior (mandibular and hyoid) arches. Likewise, transplantation of $f g f 3$ morphantderived endoderm into cas embryos only rescues the formation of the mandibular and hyoid arches but not the gills. This differential requirement for $f g f 3$ function, together with the fact that $f g f 3$ is maintained in the posterior but not the most anterior (mandibular) arch endoderm, suggests that pharyngeal endoderm may provide distinct cues along the AP axis, which could participate in the control of the formation of the distinct types of cartilage elements, together with patterning information conveyed by chondrogenic neural crest cells at the time they colonise the pharyngeal pouches.

When is Fgf3 required? Previous studies in quail have suggested a late function of FGF2 in promoting chondrogenesis (Sarkar et al., 2001). However, both the early expression of $f g f 3$ in pharyngeal endoderm and reduction of $d l x 2$ expression at $30 \mathrm{hpf}$ in $f g f 3$ morphants argue in favour of an earlier function for FGF3, in specification and survival of chondrogenic crest cells. Consistent with this idea, disruption of $f g f 8$ function in the ectoderm of the first branchial arch in mice disrupts survival of chondrogenic crest cells (Trumpp et al., 1999). FGFs can function in vitro as survival factors for a wide variety of cell types, including crest cells (reviewed by Szebenyi and Fallon, 1999). Its absence in endoderm-deprived mutants could thus directly account for the significant increase in cell death observed in these embryos.

Interestingly, whereas $f g f 3$ is necessary for posterior arch formation, it does not seem sufficient to induce these arches since transplants of cells expressing fgf3 in the pharyngeal region of cas embryos does not rescue gill arch formation nor $d l x 2$ expression (data not shown). This strongly suggests that endoderm provides other diffusible cues also required for pharyngeal arch formation, a possibility consistent with the fact that FGFs have been shown to act in synergy with other diffusible substances in head morphogenesis (Schneider et al., 2001). Alternatively, signalling from endoderm to neural crest cells might also involve additional epithelial mesenchymal interactions, including direct cell-cell contacts. Thus, an exciting challenge in the future will be to identify other cues provided by the endoderm, and the way they help organise the pharyngeal arch region.

We thank our many colleagues and the Tübingen stock center for providing plasmids and fish strains. We are indebted to L. Bally-Cuif, P. Mourrain for a critical reading of this manuscript and to G. Karsenty for suggestions regarding FGF. We thank F. Bouallague for fish care. We apologize for citing reviews rather than research articles, particularly regarding segmentation, owing to space constraints. This work was supported by grants from ARC, ACI, AFM to F. M. R. and grants from the NIH (NS-41353, DE-13828), March of Dimes (1FY01-198), Pew Scholars Foundation (2615SC) and Wellcome Trust (RCDF 055120) to T. F. S.

\section{REFERENCES}

Akimenko, M. A., Ekker, M., Wegner, J., Lin, W. and Westerfield, M. (1994). Combinatorial expression of three zebrafish genes related to distalless: part of a homeobox gene code for the head. J. Neurosci. 14, 3475-3486.

Alexander, J. and Stainier, D. Y. (1999). A molecular pathway leading to endoderm formation in zebrafish. Curr. Biol. 9, 1147-1157.

Alexander, J., Rothenberg, M., Henry, G. L. and Stainier, D. Y. (1999). casanova plays an early and essential role in endoderm formation in zebrafish. Dev. Biol. 215, 343-357. 
Aoki, T., Mathieu, J., Saint-Etienne, L., Peyriéras, N. and Rosa, F. M. (2002a). Regulation of Nodal signalling and mesendoderm formation by TARAM-A, a TGF $\beta$-related type I receptor. Dev. Biol. 241, 273-288.

Aoki, T. O., David, N. B., Minchiotti, G., Saint-Etienne, L., Dickmeis, T., Persico, G. M., Strahle, U., Mourrain, P. and Rosa, F. M. (2002b). Molecular integration of casanova in the Nodal signalling pathway controlling endoderm formation. Development 129, 275-286.

Bally-Cuif, L., Goutel, C., Wassef, M., Wurst, W. and Rosa, F. (2000). Coregulation of anterior and posterior mesendodermal development by a hairy-related transcriptional repressor. Genes Dev 14, 1664-1677.

Bruneau, S., Mourrain, P. and Rosa, F. M. (1997). Expression of contact, a new zebrafish DVR member, marks mesenchymal cell lineages in the developing pectoral fins and head and is regulated by retinoic acid. Mech. Dev. 65, 163-173.

Chen, J. N., Haffter, P., Odenthal, J., Vogelsang, E., Brand, M., van Eeden, F. J., Furutani-Seiki, M., Granato, M., Hammerschmidt, M., Heisenberg, C. P., Jiang, Y. J., Kane, D. A., Kelsh, R. N., Mullins, M. C. and Nusslein-Volhard, C. (1996). Mutations affecting the cardiovascular system and other internal organs in zebrafish. Development 123, 293-302.

Christen, B. and Slack, J. M. (1999). Spatial response to fibroblast growth factor signalling in Xenopus embryos. Development 126, 119-125.

Couly, G., Creuzet, S., Bennaceur, S., Vincent, C. and le Douarin, N. M. (2002). Interactions between Hox-negative cephalic neural crest cells and the foregut endoderm in patterning the facial skeleton in the vertebrate head. Development 129,1061-1073.

David, N. B. and Rosa, F. M. (2001). Cell autonomous commitment to an endodermal fate and behaviour by activation of Nodal signalling. Development 128, 3937-3947.

Delot, E., Kataoka, H., Goutel, C., Yan, Y. L., Postlethwait, J., Wittbrodt, J. and Rosa, F. M. (1999). The BMP-related protein radar: a maintenance factor for dorsal neuroectoderm cells? Mech. Dev. 85, 15-25.

Dickmeis, T., Mourrain, P., Saint-Etienne, L., Fischer, N., Aanstad, P., Clark, M., Strahle, U. and Rosa, F. (2001). A crucial component of the endoderm formation pathway, CASANOVA, is encoded by a novel soxrelated gene. Genes Dev. 15, 1487-1492.

Ellies, D. L., Langille, R. M., Martin, C. C., Akimenko, M. A. and Ekker, M. (1997). Specific craniofacial cartilage dysmorphogenesis coincides with a loss of dlx gene expression in retinoic acid-treated zebrafish embryos. Mech. Dev. 61, 23-36.

Feldman, B., Gates, M. A., Egan, E. S., Dougan, S. T., Rennebeck, G., Sirotkin, H. I., Schier, A. F. and Talbot, W. S. (1998). Zebrafish organizer development and germ-layer formation require nodal-related signals. Nature 395, 181-185.

Ferguson, C. A., Tucker, A. S. and Sharpe, P. T. (2000). Temporospatial cell interactions regulating mandibular and maxillary arch patterning. Development 127, 403-412.

Furthauer, M., Reifers, F., Brand, M., Thisse, B. and Thisse, C. (2001). sprouty 4 acts in vivo as a feedback-induced antagonist of FGF signaling in zebrafish. Development 128, 2175-2186.

Girdham, C. H. and O'Farrell, P. H. (1994). The use of photoactivatable reagents for the study of cell lineage in Drosophila embryogenesis. Methods Cell Biol. 44, 533-543.

Graham, A. and Smith, A. (2001). Patterning the pharyngeal arches. BioEssays 23, 54-61.

Graveson, A. C. and Armstrong, J. B. (1987). Differentiation of cartilage from cranial neural crest in the axolotl (Ambystoma mexicanum). Differentiation 35, 16-20.

Hall, B. K. (1980). Tissue interactions and the initiation of osteogenesis and chondrogenesis in the neural crest-derived mandibular skeleton of the embryonic mouse as seen in isolated murine tissues and in recombinations of murine and avian tissues. J. Embryol. Exp. Morphol. 58, 251-264.

Hauptmann, G. and Gerster, T. (1994). Two-color whole-mount in situ hybridization to vertebrate and Drosophila embryos. Trends Genet. 10, 266.

Kelsh, R. N., Dutton, K., Medlin, J. and Eisen, J. S. (2000). Expression of zebrafish fkd6 in neural crest-derived glia. Mech. Dev. 93, 161-164.

Kikuchi, Y., Agathon, A., Alexander, J., Thisse, C., Waldron, S., Yelon, D., Thisse, B. and Stainier, D. Y. (2001). casanova encodes a novel Sox-related protein necessary and sufficient for early endoderm formation in zebrafish. Genes Dev. 15, 1493-1505.

Kikuchi, Y., Trinh, L. A., Reiter, J. F., Alexander, J., Yelon, D. and Stainier, D. Y. (2000). The zebrafish bonnie and clyde gene encodes a Mix family homeodomain protein that regulates the generation of endodermal precursors. Genes Dev. 14, 1279-1289.

Kimmel, C. B., Miller, C. T., Kruze, G., Ullmann, B., BreMiller, R. A.,
Larison, K. D. and Snyder, H. C. (1998). The shaping of pharyngeal cartilages during early development of the zebrafish. Dev. Biol. 203, 245263.

Kudoh, T., Tsang, M., Hukriede, N. A., Chen, X., Dedekian, M., Clarke, C. J., Kiang, A., Schultz, S., Epstein, J. A., Toyama, R. et al. (2001). A gene expression screen in zebrafish embryogenesis. Genome Res. 11, 1979-1987.

Kurihara, Y., Kurihara, H., Suzuki, H., Kodama, T., Maemura, K., Nagai, R., Oda, H., Kuwaki, T., Cao, W. H., Kamada, N. et al. (1994). Elevated blood pressure and craniofacial abnormalities in mice deficient in endothelin-1. Nature 368, 703-710.

Launay, C., Fromentoux, V., Thery, C., Shi, D. L. and Boucaut, J. C. (1994). Comparative analysis of the tissue distribution of three fibroblast growth factor receptor mRNAs during amphibian morphogenesis. Differentiation 58, 101-111.

Le Douarin, N. M. (1982). The Neural Crest. Cambridge, UK: Cambridge University Press.

Lee, K. H., Xu, Q. and Breitbart, R. E. (1996). A new tinman-related gene, $\mathrm{nkx} 2.7$, anticipates the expression of $\mathrm{nkx} 2.5$ and $\mathrm{nkx} 2.3$ in zebrafish heart and pharyngeal endoderm. Dev. Biol. 180, 722-731.

Mahmood, R., Kiefer, P., Guthrie, S., Dickson, C. and Mason, I. (1995). Multiple roles for FGF-3 during cranial neural development in the chicken. Development 121, 1399-1410.

Marusich, M. F., Furneaux, H. M., Henion, P. D. and Weston, J. A. (1994). $\mathrm{Hu}$ neuronal proteins are expressed in proliferating neurogenic cells. $J$. Neurobiol. 25, 143-155.

Miller, C. T., Schilling, T. F., Lee, K., Parker, J. and Kimmel, C. B. (2000). sucker encodes a zebrafish Endothelin-1 required for ventral pharyngeal arch development. Development 127, 3815-3828.

Mohammadi, M., McMahon, G., Sun, L., Tang, C., Hirth, P., Yeh, B. K., Hubbard, S. R. and Schlessinger, J. (1997). Structures of the tyrosine kinase domain of fibroblast growth factor receptor in complex with inhibitors. Science 276, 955-960.

Noden, D. M. (1983). The role of the neural crest in patterning of avian cranial skeletal, connective and muscle tissues. Dev. Biol. 96, 144-165.

Odenthal, J. and Nusslein-Volhard, C. (1998). fork head domain genes in zebrafish. Dev. Genes Evol. 208, 245-258.

Pasqualetti, M., Ori, M., Nardi, I. and Rijli, F. M. (2000). Ectopic Hoxa2 induction after neural crest migration results in homeosis of jaw elements in Xenopus. Development 127, 5367-5378.

Peyrieras, N., Strahle, U. and Rosa, F. (1998). Conversion of zebrafish blastomeres to an endodermal fate by TGF-beta-related signaling. Curr. Biol. 8, 783-786.

Phillips, B. T., Bolding, K. and Riley, B. B. (2001). Zebrafish fgf3 and fgf8 encode redundant functions required for otic placode induction. Dev. Biol. 235, 351-365.

Piotrowski, T. and Nusslein-Volhard, C. (2000). The endoderm plays an important role in patterning the segmented pharyngeal region in zebrafish (Danio rerio). Dev. Biol. 225, 339-356.

Reissmann, E., Jornvall, H., Blokzijl, A., Andersson, O., Chang, C., Minchiotti, G., Persico, M. G., Ibanez, C. F. and Brivanlou, A. H. (2001). The orphan receptor ALK7 and the Activin receptor ALK4 mediate signaling by Nodal proteins during vertebrate development. Genes Dev. 15, 2010-2022.

Roehl, H. and Nusslein-Volhard, C. (2001). Zebrafish pea3 and erm are general targets of FGF8 signaling. Curr. Biol. 11, 503-507.

Sarkar, S., Petiot, A., Copp, A., Ferretti, P. and Thorogood, P. (2001). FGF2 promotes skeletogenic differentiation of cranial neural crest cells. Development 128, 2143-2152.

Schier, A. F. and Shen, M. M. (2000). Nodal signalling in vertebrate development. Nature 403, 385-389.

Schilling, T. F. (1997). Genetic analysis of craniofacial development in the vertebrate embryo. BioEssays 19, 459-468.

Schilling, T. F. and Kimmel, C. B. (1994). Segment and cell type lineage restrictions during pharyngeal arch development in the zebrafish embryo. Development 120, 483-494.

Schilling, T. F. and Kimmel, C. B. (1997). Musculoskeletal patterning in the pharyngeal segments of the zebrafish embryo. Development 124, 29452960.

Schilling, T. F., Piotrowski, T., Grandel, H., Brand, M., Heisenberg, C. P., Jiang, Y. J., Beuchle, D., Hammerschmidt, M., Kane, D. A., Mullins, M. C., van Eeden, F. J., Kelsh, R. N., Furutani-Seiki, M., Granato, M., Haffter, P., Odenthal, J., Warga, R. M., Trowe, T. and Nusslein-Volhard, C. (1996b). Jaw and branchial arch mutants in zebrafish I: branchial arches. Development 123, 329-344. 
Schilling, T. F., Prince, V. and Ingham, P. W. (2001). Plasticity in zebrafish hox expression in the hindbrain and cranial neural crest. Dev. Biol. 231, 201216.

Schilling, T. F., Walker, C. and Kimmel, C. B. (1996a). The chinless mutation and neural crest cell interactions in zebrafish jaw development. Development 122, 1417-1426.

Schneider, R. A., Hu, D., Rubenstein, J. L., Maden, M. and Helms, J. A. (2001). Local retinoid signaling coordinates forebrain and facial morphogenesis by maintaining FGF8 and SHH. Development 128, 27552767.

Seufert, D. W. and Hall, B. K. (1990). Tissue interactions involving cranial neural crest in cartilage formation in Xenopus laevis (Daudin). Cell Differ. Dev. 32, 153-165.

Shinya, M., Koshida, S., Sawada, A., Kuroiwa, A. and Takeda, H. (2001). Fgf signalling through MAPK cascade is required for development of the subpallial telencephalon in zebrafish embryos. Development 128, 4153 4164.

Stainier, D. Y., Fouquet, B., Chen, J. N., Warren, K. S., Weinstein, B. M., Meiler, S. E., Mohideen, M. A., Neuhauss, S. C., Solnica-Krezel, L., Schier, A. F., Zwartkruis, F., Stemple, D. L., Malicki, J., Driever, W. and Fishman, M. C. (1996). Mutations affecting the formation and function of the cardiovascular system in the zebrafish embryo. Development 123, 285292

Strahle, U., Blader, P., Henrique, D. and Ingham, P. W. (1993). Axial, a zebrafish gene expressed along the developing body axis, shows altered expression in cyclops mutant embryos. Genes Dev. 7, 1436-1446.

Szebenyi, G. and Fallon, J. F. (1999). Fibroblast growth factors as multifunctional signaling factors. Int. Rev. Cytol. 185, 45-106.

Tickle, C. and Munsterberg, A. (2001). Vertebrate limb development-the early stages in chick and mouse. Curr. Opin. Genet. Dev. 11, 476-481.
Trainor, P. A. and Krumlauf, R. (2001). Hox genes, neural crest cells and branchial arch patterning. Curr. Opin. Cell Biol. 13, 698-705.

Trainor, P. A. and Tam, P. P. (1995). Cranial paraxial mesoderm and neural crest cells of the mouse embryo: co-distribution in the craniofacial mesenchyme but distinct segregation in branchial arches. Development 121, 2569-2582.

Trevarrow, B., Marks, D. L. and Kimmel, C. B. (1990). Organization of hindbrain segments in the zebrafish embryo. Neuron 4, 669-679.

Trumpp, A., Depew, M. J., Rubenstein, J. L., Bishop, J. M. and Martin, G. R. (1999). Cre-mediated gene inactivation demonstrates that FGF8 is required for cell survival and patterning of the first branchial arch. Genes Dev. 13, 3136-3148.

Veitch, E., Begbie, J., Schilling, T. F., Smith, M. M. and Graham, A. (1999). Pharyngeal arch patterning in the absence of neural crest. Curr. Biol. 9, 1481-1484.

Wall, N. A. and Hogan, B. L. (1995). Expression of bone morphogenetic protein-4 (BMP-4), bone morphogenetic protein-7 (BMP-7), fibroblast growth factor-8 (FGF-8) and sonic hedgehog (SHH) during branchial arch development in the chick. Mech. Dev. 53, 383-392.

Warga, R. M. and Nusslein-Volhard, C. (1999). Origin and development of the zebrafish endoderm. Development 126, 827-838.

Wendling, O., Dennefeld, C., Chambon, P. and Mark, M. (2000). Retinoid signaling is essential for patterning the endoderm of the third and fourth pharyngeal arches. Development 127, 1553-1562.

Yan, Y. L., Jowett, T. and Postlethwait, J. H. (1998). Ectopic expression of hoxb2 after retinoic acid treatment or mRNA injection: disruption of hindbrain and craniofacial morphogenesis in zebrafish embryos. Dev. Dyn. 213, 370-385.

Yeo, C. and Whitman, M. (2001). Nodal signals to Smads through Criptodependent and Cripto-independent mechanisms. Mol. Cell 7, 949-957. 